\title{
Application of Poensgen apparatus for determining the thermal conductivity of reflective insulations
}

\author{
Bartosz Radomski ${ }^{1, *}$, and Lawrence Drojetzki ${ }^{1}$ \\ ${ }^{1}$ Poznan University of Technology, Institute of Environmental Engineering, Poznan, Poland
}

\begin{abstract}
Multilayer reflective insulations are a combination of several or a dozen low emission screens compressed together, separated by layers of bubble foil or polyethylene foam. This paper presents the results of experimental investigation of thermal conductivity of multilayer reflective insulations. The measurements were made with Poensgens LaserComp FOX 314 apparatus. Five samples of reflective insulation and one sample of foamed polystyrene have been tested. The difficulties of determining the replacement thermal resistance for the discussed materials have been presented. Information about the apparatus used, theory of the measurement method, procedure of the experiment and method of sample preparation were presented. The study was performed for four different temperature pairs representing the real thermal conditions in Poland throughout the year. On the basis of the obtained measurement results the average thermal conductivity and average thermal resistance of the tested materials were determined. The relation between the obtained thermal conductivity and thermal resistance depending on the temperature was discussed.
\end{abstract}

\section{Introduction}

Multilayer reflective insulation is a combination of several or dozen low emission screens (about $0.01 \mathrm{~mm}$ thick) pressed together (Fig. 1). Mostly aluminium foil is used. The foil layers are separated by bubble foil or polyethylene foam. Those fillings are an additional element increasing thermal insulation properties $[1,2]$. The use of several layers of the foil results in higher total thermal resistance than the use of the same thickness of insulation with fewer layers [1,2]. According to the literature, reflective foils and mats require less space in the building envelope than traditional thermal insulations. As a result, they provide an interesting alternative to the commonly used styrofoam (foamed polystyrene) or mineral wool.

\footnotetext{
*Corresponding author: bartosz.an.radomski@doctorate.put.poznan.pl
} 


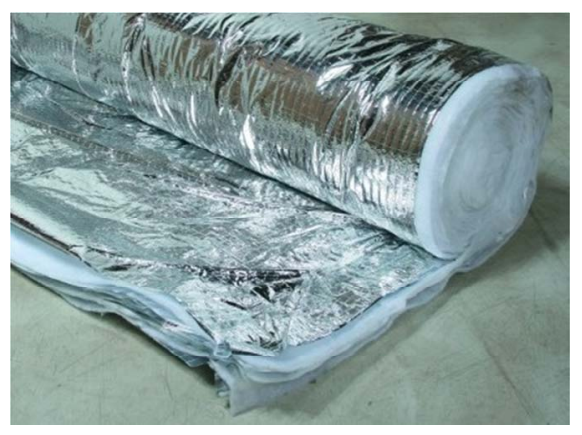

Fig. 1. Multilayer reflective insulation.

Presented properties of multilayer reflective insulations persuaded the authors to verify their thermal resistance experimentally. It was decided to test the materials with Poensgen apparatus and the results are presented in this paper.

\section{Difficulties in determining replacement thermal resistance}

The idea of reflective insulation is to minimize the absorption of heat transferred by radiation. Significant heat transfer resistance resulting from the use of reflective foil can be achieved only by mounting the foil in the air gap. In the studies $[1,8]$ it was shown that air space on both sides has a significant impact on the thermal resistance of reflective mats in building partitions. In the literature [1] and in the manufacturers' brochures, the thickness of the air gaps of 10-20 mm is indicated as a minimum. The study [8] showed that there is a correlation between the thickness of air gaps and the total thermal resistance of a partition using reflective mats. As the thickness of the air layers increases, the total thermal resistance of the partition also rises and the space of $19 \mathrm{~mm}$ is indicated as the optimum. It should be noted that larger gaps may cause convective air flows which, by intensifying convective heat transfer, would reduce the thermal resistance of the structure. The samples analyzed by the authors have air gaps of $40 \mathrm{~mm}$ thickness on both sides.

Convection compared to conduction is a more complex phenomenon. It depends on the specific heat transfer parameters that vary from case to case. The dimensions of the wall and of the air gap influence the convective heat flow. The direction of heat conduction and the orientation of the barrier are of significance. A different description is used for the heat flux penetrating the horizontal partition and a different one through the vertical envelope. Account should be taken of whether or not the air gap is ventilated. For natural convection, heat transfer coefficients can vary considerably across the heating season. The phenomenon that causes this type of heat exchange is the difference in temperature of the surface of the barrier and the air in contact with it. The mentioned temperature differences depend on weather changes. This dependency significantly affects the intensity of heat transfer, thus influence thermal parameters of the partition. For forced convection, the velocities of the air that flushes the surface of the reflective mat are important for the ventilated air gap.

The multiplicity of factors influencing the heat exchange through partitions containing reflective mats causes that reliable values of replacement thermal resistance of such partitions can be obtained only experimentally. In the 1980s, research was conducted with the use of a calibrated hot box device, which resulted in the conclusion that the measuring devices of this type (guarded hot box and guarded hot plates) were inaccurate. The reason for this is that they measure heat transfer only through the centre of the test material. The measuring range varies from instrument to instrument. The Poensgen apparatus used by the authors has a measuring area of $10 \times 10 \mathrm{~cm}$, located in the middle of the plates. Measurement with these types of devices may be less accurate than with a calibrated hot 
box. The results may be underestimated [1]. Despite the detected inaccuracies, according to Miller [7] the heat flow meter is suitable for measuring the thermal resistance of reflective materials. Nonetheless, it is recommended that the air gaps do not exceed $50 \mathrm{~mm}$ for the measurement method used in this way [9]. It is therefore appropriate to use the Poensgens apparatus - LaserComp FOX 314, which is a heat flow meter.

\section{Measurements}

\subsection{Poensgen apparatus}

To determine the replacement thermal resistance of multilayer reflective insulations, a Poensgen apparatus (Fig. 2) LaserComp FOX 314 was used. The device was developed in accordance with ASTM C518-91 [3] and the results of the thermal conductivity measurements are calculated in accordance with ASTM C1045-90 [4]. The apparatus consists of a measuring chamber, which is equipped with two plates with a heating and cooling system (Fig. 3). The upper one is permanently installed and the lower one is used to press the sample. Each plate has a built-in high-sensitivity heat flux transmitter consisting of thousands of small thermocouples. The signals from the heat flux transmitters are proportional to the heat flux flowing through the tested sample and accurately convey information about the thermal conductivity of the sample [5].

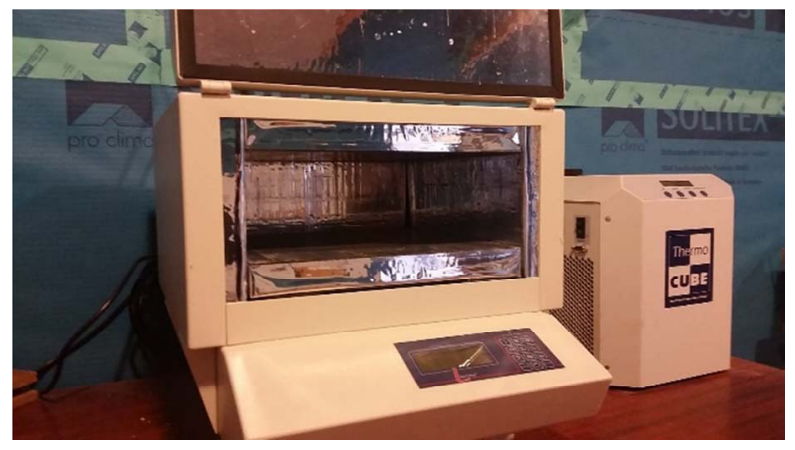

Fig. 2. Poensgen apparatus - LaserComp FOX 314.

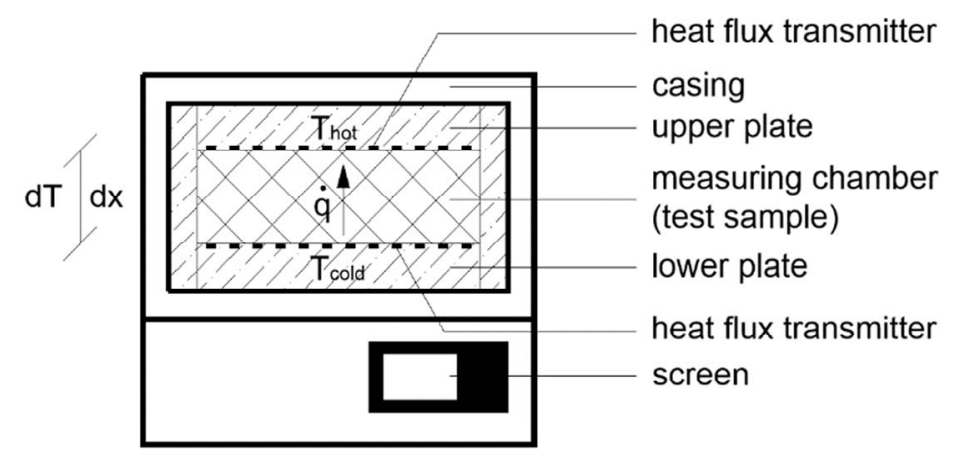

Fig. 3. Scheme of Poensgen device. 


\subsection{Criterion}

Specification of the measuring instrument used:

- Measuring range of thermal conductivity $k$

- Accuracy of measurement

- Repeatability of measurement

- Reproducibility of measurement

- Maximal hot plate temperature

- Minimal cold plate temperature

- Stability of the maintained temperature

- Accuracy of specimens thickness measurement

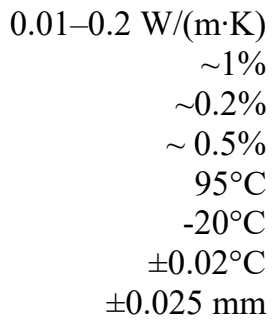

Permissible sample sizes:

- Maximal in plan

- Minimal in plan

- Maximal thickness

- Measuring area

$$
\begin{array}{r}
305 \times 305 \mathrm{~mm} \\
150 \times 150 \mathrm{~mm} \\
100 \mathrm{~mm} \\
100 \times 100 \mathrm{~mm}
\end{array}
$$

Laboratory measurements are performed in steady state conditions. The conditions on both sides of the partition are simulated in the chamber. The Poensgen apparatus cannot simulate the solar influence on the thermal insulation properties of a material. Hence the efficiency of reflective mats in the summer season cannot be obtained. Another imperfection of the measurements is the inability to take into account the variable air humidity on both sides of the reflective barrier [1].

\subsection{Measurement method}

The FOX type test device is based on the application of the one-dimensional Fourier-Biot law (Eq. 1).

$$
q=-k(d T / d x)
$$

Where:

$q\left[\mathrm{~W} / \mathrm{m}^{2}\right]$ - heat flux density flowing through the sample, $k[\mathrm{~W} /(\mathrm{m} \cdot \mathrm{K})]-$ thermal conductivity of the sample, $d T / d x[\mathrm{~K} / \mathrm{m}]$ - temperature gradient.

By placing the test sample between two flat isothermal plates with two different imposed temperatures, the sample strives to obtain a homogeneous one-dimensional temperature field over the entire volume of the sample. The temperature gradient may then be determined by measuring the temperature difference between the plates $\left(\Delta \mathrm{T}=\mathrm{T}_{\text {hot }}-\mathrm{T}_{\text {cold }}\right)$ and the thickness of the sample $(\Delta \mathrm{x})$. In this situation the mean temperature gradient $\mathrm{dT} / \mathrm{dx}$ is equal to $-\Delta \mathrm{T} / \Delta \mathrm{x}$.

Thermal conductivity of the sample is calculated for each plate and for each temperature pair separately. The final outcome for each temperature pair is calculated as an average of the values obtained for the individual plates.

To ensure correct measurement three criteria must be met: temperature, semicompliance and percentage. The temperature criterion is verified first. The average temperature of each of the plates in the measuring block must not deviate from the set temperature by more than the set criterion (default: $0.2^{\circ} \mathrm{C}$ ). The semi-compliance criterion states that the difference between the mean values of the transmitter signals in two following measurement blocks cannot exceed the set value of the criterion (default: $49 \mu \mathrm{V}$ ). The last criterion to be verified is the percentage criterion. Its fulfilment consists of the requirement that the average signal value of the transmitters in two subsequent measurement blocks must be in the range defined by that criterion. Default - the maximum 
difference between the measured heat flux values on the upper plate $\left(\mathrm{Q}_{\mathrm{U}}\right.$ avrg$)$ in two following blocks and on the lower plate $\left(\mathrm{Q}_{\mathrm{L} \text { avrg }}\right)$ in two consecutive blocks is $2 \%$. If four following measurement blocks meet the percentage criterion and the $\mathrm{Q}_{\text {avrg }}$ for the subsequent measurement blocks do not change in one direction, the measurement is deemed correct and the result of the measurement is recorded [5].

\subsection{Samples of the tested materials}

Samples of the tested materials were prepared carefully. The multilayer reflective films were placed in specially prepared measuring boxes (Fig. 4) with dimensions $300 \times 300 \mathrm{~mm}$ in the plan and the desired height of approx. $90 \mathrm{~mm}$. The boxes are made of squared timber $40 \mathrm{~mm}$ high and $19 \mathrm{~mm}$ thick. The sample edges that come into direct contact with the instruments plates were covered with a window seal to prevent air leakage out of the sample space. The gaps were filled with silicone, which ensured a sufficient air tightness. In order to ensure the stability, durability and appropriate size of the measuring boxes, they were stiffened with steel angles (Fig. 5).

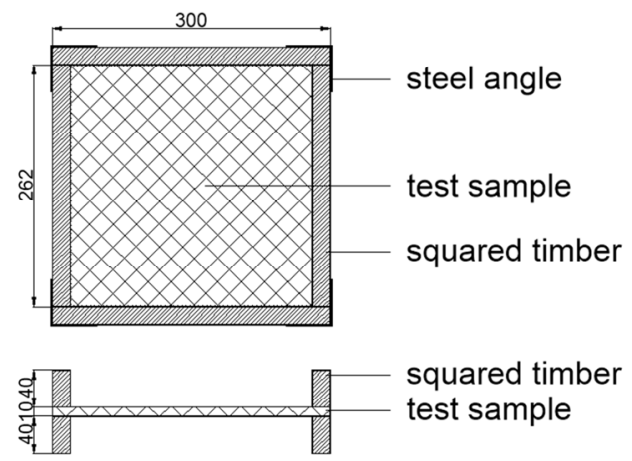

Fig. 4. A throw and cross-section of the tested measuring boxes.

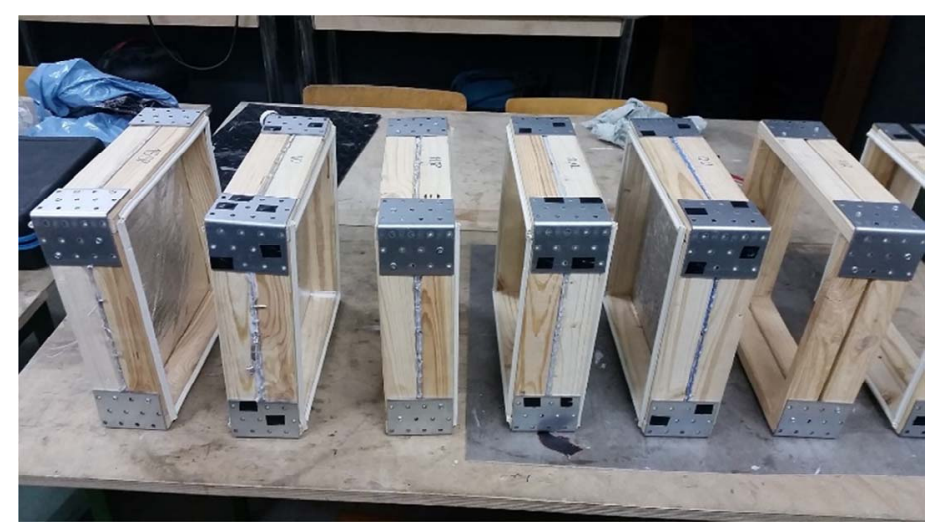

Fig. 5. Tested measuring boxes with multi-layer reflective insulation.

\section{Results}

The first step in the measurement process was to calibrate the Poensgen instrument. Afterwards the thickness of the test sample was measured. Then the temperature pairs for the upper and lower plate were determined and input. A constant temperature difference of 
$\Delta \mathrm{T}=25^{\circ} \mathrm{C}$ was assumed. The measurements were carried out for four temperature pairs. The sample was tested in a horizontal orientation. The heat flux direction was upward. A single test for one temperature pair for the assumed thickness of the sample with a reflective mat lasted about 4-8 h. The complete test for one sample took at least 16-24 h. After the test, the calculated values of the thermal conductivity coefficient were displayed on the screen. Results have been given separately for each plate and for each temperature pair. All data and outcomes are collected in Table 1. and presented in the form of a graph showing the dependence of the determined thermal conductivity and thermal resistance on the temperature of the measurement plates (Fig. 6). The material tested in the sample No. 6 was foamed polystyrene with known parameters. It was tested to verify the influence of the enclosure on the result of thermal conductivity of the sample. Obtained thermal diffusivity of the styrofoam was similar with the expected one, therefore it can be induce that the disturbing influence of the box was negligible. The measurements were carried out in steady state conditions for the imposed thermal conditions on both sides of the tested partition.

As shown in Fig. 6, the thermal conductivity coefficient (solid lines) and the thermal resistance (dashed lines) are temperature dependent. With the increase in temperature the thermal conductivity coefficient rises, while the thermal resistance decreases.

Due to the unexpected results of testing of samples containing reflective insulations with air gaps, supplementary measurements were made. For comparison, two reflective insulations were tested with the apparatus without any air gap for the same four temperature pairs. In the case of the sample No. 2, the average thermal conductivity coefficient was obtained equal to $30.93 \mathrm{~mW} /(\mathrm{m} \cdot \mathrm{K})$. The result for the sample No. 3 was lower and reached the value of $28.3 \mathrm{~mW} /(\mathrm{m} \cdot \mathrm{K})$.

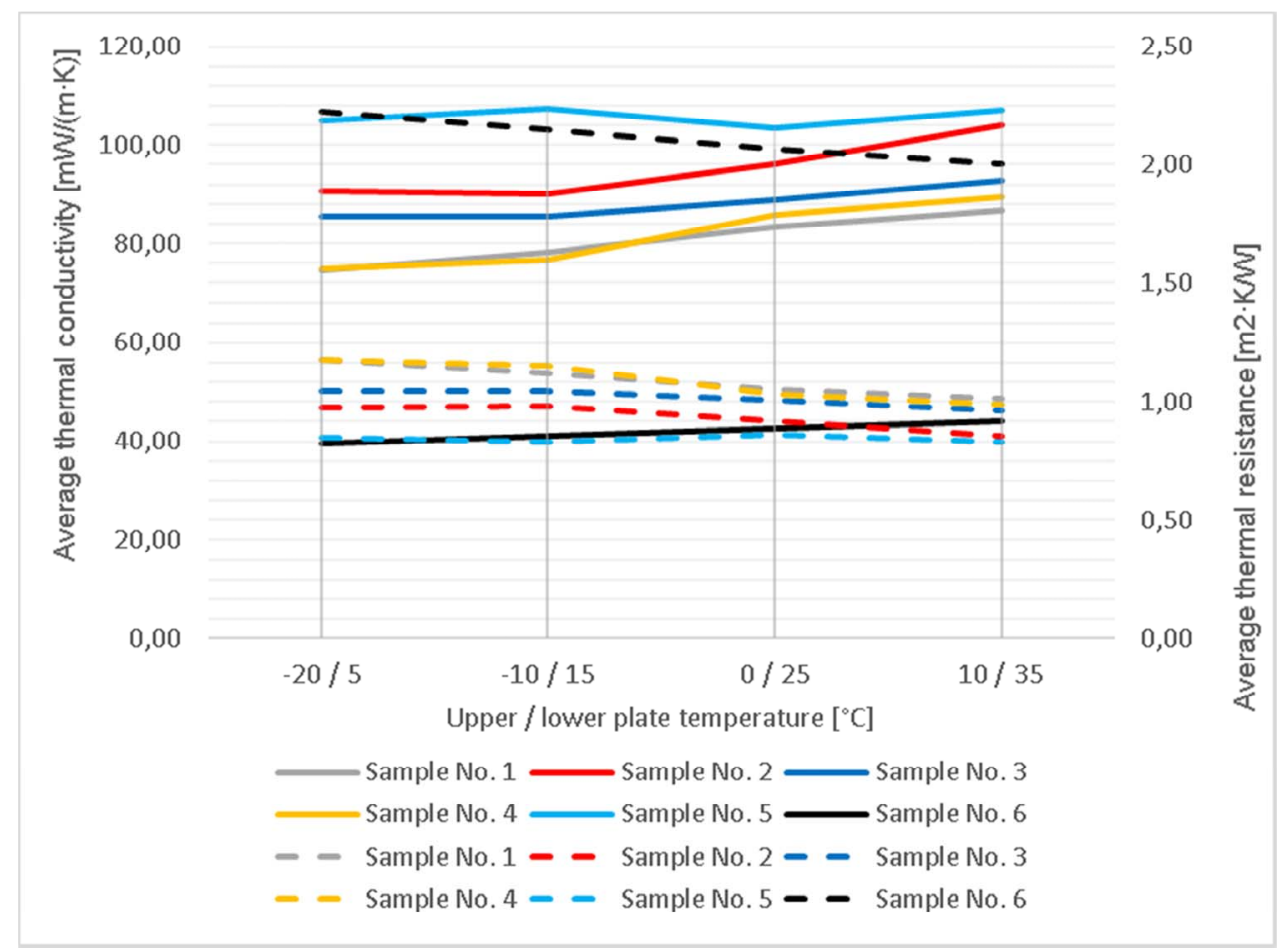

Fig. 6. The dependence of the determined thermal conductivity and thermal resistance on the temperature of the measuring plates. 


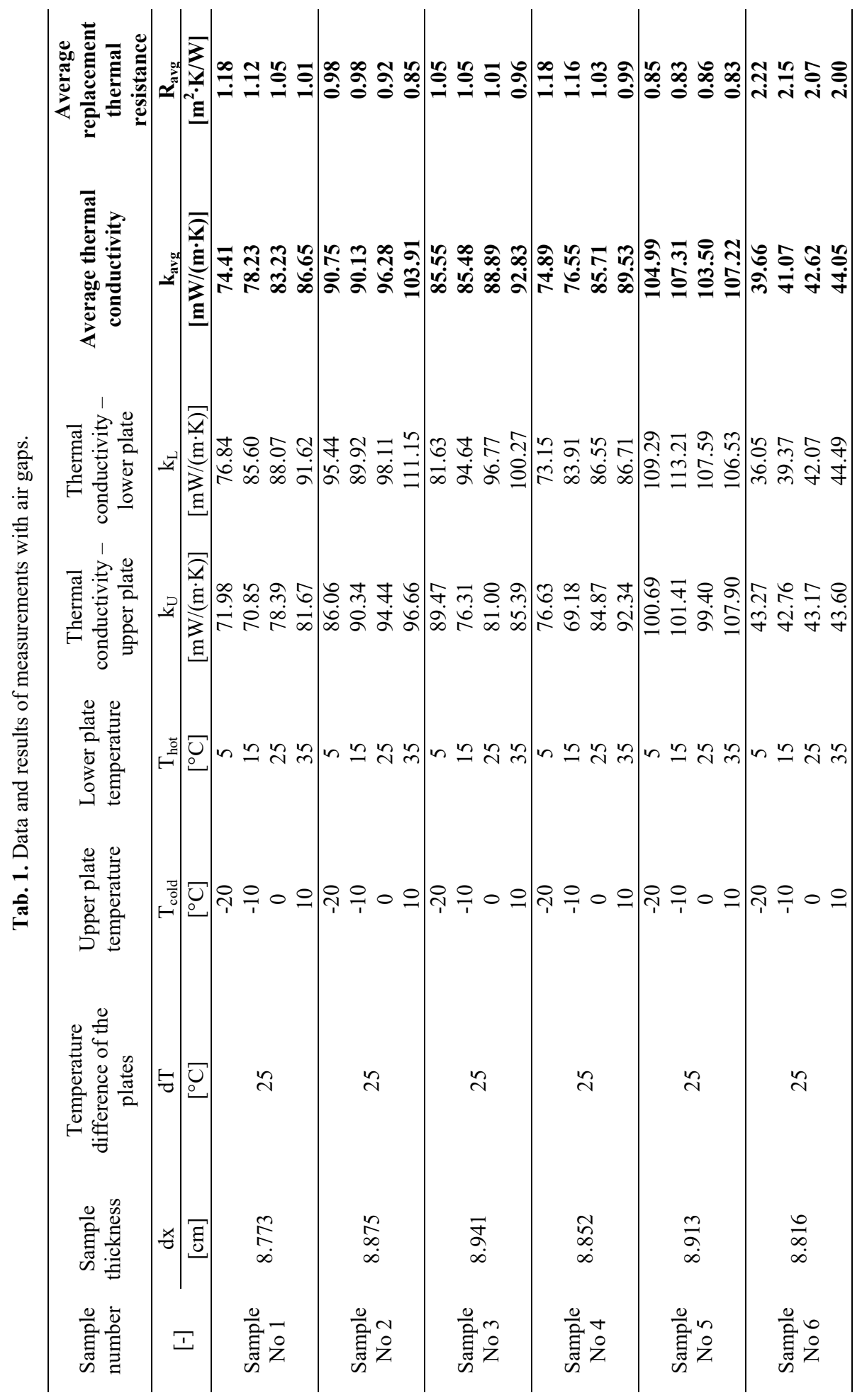




\section{Conclusions}

Due to their comparable properties and price, multilayer reflective insulations are an alternative to traditional insulation materials. These materials themselves have lower thermal conductivity and higher thermal resistance. The studies have shown that the thermal conductivity of the tested reflective insulations ranges between 0.028 and $0.031 \mathrm{~W} /(\mathrm{m} \cdot \mathrm{K})$, which places them slightly above foamed polystyrene and mineral wool. However, the tested construction of reflective mats together with air gaps on both sides, in accordance with the obtained measurement results, is characterized by higher thermal conductivity, i.e. lower thermal resistance than traditional insulating materials at the same thickness of the external partition.

Application of the Poensgen apparatus for determining the thermal conductivity of reflective insulations in gives a substantially reliable results. An inadequacy of this method of measurement is the inability to take into account variable relative humidity on both sides of the partition. A significant limitation is that it is not possible to test the reduction of solar heat gains in summer and the radiant heat dissipation in winter. In order to obtain a complete overview and to know the real effectiveness of the application of reflective insulations, the materials should be applied in a real building and examined in-situ throughout the year.

Referring to the obtained experimental results and the untested but known properties of the reflective mats, it can be concluded that the use of this type of insulation in combination with traditional materials can ensure good thermal and moisture results of the partition.

\section{References}

1. S.W. Lee, Ch.H. Lim, E. Bin Salleh, Renewable and Sustainable Energy Reviews. 65 (2016)

2. R. Wójcik, Izolacje 11/12 (2014)

3. ASTM C518-17: Standard Test Method for Steady-State Thermal Transmission Properties by Means of the Heat Flow Meter Apparatus

4. ASTM C1045-07(2013): Standard Practice for Calculating Thermal Transmission Properties Under Steady-State Conditions

5. LaserComp FOX314: Instrukcja obstugi. PARSER Sp. z o.o.

6. Reflective Insulation. Radiant Barriers and Radiation Control Coatings, RIMA, 2002.

7. R.G. Miller, D.S. Oscar, F. Seifaee, W.P. Goss, Conf. Therm. Insul. Mater. Test. Appl. (1987)

8. E.R. Queer, ASHVE Trans 38 (1932)

9. D.W. Yarbrough, Materials for Energy Efficiency and Thermal Comfort in Buildings, (Woodhead Publishing, Sawston, 2010) 